\title{
Des Edge-Cities en Suisse? L'émergence de nouveaux pôles d'activités métropolitaines sur le territoire helvétique
}

\section{Pierre Dessemontet, Lausanne}

\section{Introduction}

Dans une précédente contribution (DESSEMONTET \& RACINE I996), nous avions pu remarquer que les attributs traditionnels de la centralité débordaient les limites des centres de certaines agglomérations suisses. En Amérique du Nord a atteint une telle dimension que les distinctions traditionnellement ancrées sur l'opposition centrepériphérie semblent aujourd'hui caduques. Il nous a donc semblé qu'il était temps de consacrer une étude au développement des activités dites centrales dans les périphéries urbaines suisses. Les conditions de développement des activités tertiaires dans les périphéries urbaines étant très différentes en Suisse et en Amérique du Nord, notre but consiste ici à repérer les régions du pays où de nouveaux centres de service en périphérie se mettent en place, et ce même si ni la taille des formes créées, ni leur stade de développement ne correspondent aux standards nord-américains, en proposant à cette fin un certain nombre d'outils méthodologiques.

\subsection{Banlieues, mutations urbaines et déconcentrations}

Depuis la vague d'urbanisation industrielle de nos sociétés, nous nous représentons généralement les systèmes intra- et interurbains selon le modèle opposant centre et périphérie. Ce modèle décrit la ville comme un centre cumulant l'essentiel des fonctions urbaines et dominant le territoire alentour, ainsi que comme un centre-ville économiquement dominant entouré de faubourgs résidentiels. Ce modèle a rendu d'éminents services aux géographes dans leur effort pour rendre compte des phénomènes d'urbanisation et de structuration intra-urbaine pendant près d'un siècle. On le retrouve dans une grande partie des écrits actuels portant sur la structure interne de la ville. Pourtant, tout porte à croire que ce modèle ne permet plus de représenter leur morphogenèse.

Deux phénomènes ont en effet remis en cause le modèle. Premièrement, le fait que, depuis cinquante ans, la ville a vécu un puissant processus de déconcentration. Le premier de ces processus fut celui de la suburbanisation, à savoir le débordement de la ville hors de ses limites, en suivant une stricte continuité territoriale et en créant un paysage clairement urbain: les couronnes internes. Le deuxième bouleversement, suivant l'amélioration radicale des moyens de transports, fut celui de la périurbanisation, étalement beaucoup plus lâche du noyau urbain vers ses périphéries, associé à la formation des agglomérations urbaines extensives et intégrées telles que nous les connaissons actuellement (BASSAND, LERESCHE \& JOYE 1995). Le phénomène de métropolisation est plus récent. Depuis quelques années, on voit apparaître une nouvelle classe urbaine, qu'on nommera métropoles, qui ont comme caractéristique fondamentale leur intégration dans l'économie-monde. Cette intégration se manifeste géographiquement par l'abandon de la logique de proximité et de centralité, au profit de contacts toujours plus développés entre noyaux métropolitains. Il se forme en outre un réseau mondial de métropoles, ces dernières «sortant» de leur territoire, abandonnant en partie les logiques ville-campagne au profit des logiques de réseau. De telles métropoles cumulent les fonctions centrales de l'économie: présence de sièges sociaux de multinationales, présence extensive de la $R \& D$, excellence du savoir-faire technologique entre autres (BASSAND, LeRESCHE \& JOYE 1995). Le lieu géographique nouveau où ces deux phénomènes (métropolisation et déconcentration des activités tertiaires) se marquent le mieux, parce qu'ils s'y superposent, est l'Edge-City.

\subsection{Edge-City}

Une Edge-City, au sens où nous l'entendrons dans cet article, est donc une zone de la périphérie urbaine où, en quelques années, viennent se concentrer des activités traditionnellement centrales. Autour d'un centre commercial généralement massif et diversifié s'établissent des centres d'entreprises d'importance internationale, abritant de nombreux emplois qualifiés dans le tertiaire et le tertiaire supérieur, ainsi que des entreprises innovantes dans les domaines des services aux entreprises, de la haute technologie, de l'information. Ce noyau est accompagné par toute la gamme de services dérivés que son apparition entraîne: hôtels, restaurants, services personnels. En termes de fonctions, l'Edge-City est une réplique du centreville, avec ses fonctions complètes, mais située en périphérie, «au bord» de la ville, d'où son nom anglo-saxon (ville-limite, ville-bord).

En 1991, J. GarREAu publia un ouvrage de référence exclusivement consacré à l'émergence des EdgeCities, qui mit à jour les règles empiriques qui régissent leur développement. Quelques articles spécialisés ont formalisé ces découvertes pour le 
compte de la recherche en géographie urbaine (BERRY \& Kim 1993). Il reste que l'état de la recherche scientifique consacrée au développement des Edge-Cities et à leurs implications sur la structure urbaine est encore embryonnaire, et que l'essentiel des études menées sur ce thème se sont focalisées sur les mégapoles nord-américaines. Or, comme le constate J. VANCE dans un ouvrage de référence, du point de vue de la morphologie de nos villes, l'écart n'a cessé de se creuser depuis le début du siècle entre les villes américaines et les villes européennes (VANCE 1990). Il y a donc ici un territoire largement vierge dans la recherche urbaine européenne. Le sujet est d'autant plus passionnant que pour la première fois dans l'histoire urbaine depuis très longtemps, nous avons affaire à la création d'une forme urbaine majeure qui ne semble répondre à aucun projet politique et urbanistique conscient et formulé: une création sans plan préétabli, sans modèle à suivre, une génération spontanée.

\subsection{Le cas suisse}

Il est bien établi que les mutations urbaines apparaissent plus rapidement en Amérique du Nord qu'en Europe, et qu'elles s'y développent avec plus de puissance. Par ailleurs, la Suisse n'abrite qu'une petite métropole de niveau mondial (Zurich), entourée d'un dense réseau de villes moyennes. Dès lors, le phénomène des Edge-Cities ne s'y développe pas selon les mêmes modalités qu'en Amérique du Nord, où il est surtout documenté aux abords des mégapoles d'importance mondiale. On peut s'attendre à ne pas trouver en Suisse beaucoup d'Edge-Cities au sens américain du terme, mais ceci ne signifie pas qu'il ne se passe rien dans les périphéries urbaines. Des études ont déjà rendu compte des nouvelles fonctions urbaines dévolues à certaines communes de banlieue. Ainsi, M. Schuler (1993) intègre-t-il certaines communes de banlieue dans les noyaux des agglomérations qu'il définit. En regard de notre objectif, plus ciblé, quels outils méthodologiques utiliser?

\section{Méthodologie}

\subsection{La définition de Garreau}

J. GarReau a le mérite de poser une définition simple permettant de détecter statistiquement une Edge-City. Selon cette définition, une Edge-City regroupe au moins:

- 25000 emplois de bureau,

- $50000 \mathrm{~m}^{2}$ de surfaces de vente

(équivalant à un gros centre commercial),

- plus de places de travail que d'actifs résidants.

De plus, elle doit intégrer de multiples fonctions et doit s'être développée hors des centres-villes à un rythme rapide, tout en étant perçue comme un lieu. Une définition qui laisse une certaine marge d'interprétation, tant le phénomène peut prendre des formes multiples.

\subsection{Pour une définition des Edge-Cities en Suisse}

Nous postulons que pour l'essentiel, les EdgeCities suisses sont encore en phase de développement: la composante dynamique occupera donc une place centrale dans la méthode de détection que nous proposons. Par ailleurs, c'est la croissance globale du secteur tertiaire qui nous intéressera ici, étant entendu que le départ du phénomène est souvent donné par le développement d'activités commerciales (Garreau 1991). Nous nous y tiendrons en laissant pour plus tard le problème développement d'activités tertiaires supérieures. Enfin, le seuil de taille défini par J. Garreau n'est pas un seuil absolu, et des formes analogues à celles des Edge-Cities peuvent se mettre en place à une échelle plus réduite.

Afin de reprendre au mieux les caractéristiques des Edge-Cities nord-américaines, cette croissance tertiaire doit en principe être le fait du secteur privé, et ne pas être liée aux activités touristiques.

\subsection{Méthode de détection}

Nous nous inspirons fortement du type de démarche mise en place par M. Schuler dans le but de définir les agglomérations suisses. L'unité statistique de notre recherche est la commune; les données statistiques sur lesquelles nous travaillons viennent des recensements des entreprises 1975, 1985, 1991 et 1995, ainsi que du recensement fédéral de la population de 1990, les données ayant été regroupées selon le découpage communal 1996. Le schéma général de notre méthode de détection est le suivant:

- Détecter, parmi l'ensemble des communes suisses, les communes concernées en les sélectionnant à l'aide de différents critères.

- Sur la base de cette sélection, regrouper les communes géographiquement contiguës en ensembles, lorsqu'elles apparaissent fonctionnellement intégrées.

- Rechercher, à la périphérie des ensembles ainsi constitués, des communes n'ayant pas été sélectionnées, mais qui n'altèrent pas l'ensemble et paraissent connaître la même dynamique, afin de les y intégrer dans un second temps.

On comprendra qu'une telle démarche laisse une grande liberté d'interprétation. Notre but étant ici de proposer une liste indicative, il s'agira de l'envisager à sa juste valeur: nous ne prétendons pas donner une liste exhaustive et définitive des 
communes non centrales connaissant un phénomène de croissance des activités tertiaires, mais bien de proposer des pistes de recherche.

\subsection{Critères de détection}

Afin d'être retenue, une commune doit satisfaire aux critères suivants:

1. Compter au moins 200 emplois à plein-temps dans le secteur tertiaire en 1995.

2. Avoir connu, lors des deux périodes $1975-1985$ et 1985-1995, une croissance des emplois tertiaires à plein-temps supérieure de 10 points à la croissance moyenne suisse (resp. de $13,8 \%$ et de $8,3 \%$ ). Si la croissance a été très supérieure à ce seuil lors d'une des deux périodes, elle peut avoir été moindre durant l'autre période.

3. En 1995, la part des emplois dans l'hôtellerierestauration, ainsi que dans les secteurs de la santé et de l'enseignement, ne doit pas excéder chacune plus de $25 \%$ du total des emplois tertiaires; regroupés, ils ne doivent pas excéder plus du tiers du total des emplois tertiaires, sauf si la croissance totale des emplois tertiaires ne peut être expliquée par la seule croissance de ces secteurs.

4. La commune doit compter plus d'emplois tertiaires à plein-temps que de résidants actifs dans le tertiaire, ou se rapprocher rapidement de ce seuil (forte croissance des emplois tertiaires).

Enfin, un seuil minimal a été fixé à 500 emplois à plein-temps pour les ensembles constitués.

Le premier critère vise à ne sélectionner que les communes connaissant une activité significative dans le tertiaire; lors de la phase 3 de la démarche, il est possible d'incorporer à un ensemble une petite commune qui satisferait aux autres critères. Le second critère est central. Il vise à sélectionner les communes connaissant une forte croissance de leurs activités tertiaires lors des vingt dernières années; de ce fait, il élimine en principe les centres historiques des agglomérations. Le phénomène pouvant se manifester à différentes époques selon les cas, il nous a semblé utile de ne pas systématiquement éliminer les communes qui ne répondent pas au critère lors d'une des deux périodes. Le troisième critère vise à exclure les communes à dominante touristique, ou celles connaissant un développement dû aux collectivités publiques. $\mathrm{Ce}$ critère est également à interpréter: il peut arriver qu'une commune voie s'implanter une telle infrastructure tout en connaissant une croissance soutenue des activités tertiaires privées. Le dernier critère vise à regrouper les communes dans lesquelles l'importance des activités tertiaires est telle qu'elle est devenue un centre d'emploi tertiaire plutôt qu'une zone résidentielle, ou qu'elle s'en approche.

\section{Résultats}

\subsection{Remarques préliminaires}

Les différents critères de sélection ont retenu 177 communes se regroupant en 61 ensembles. La liste de ces unités territoriales est donnée dans le tableau 1, par ordre de «taille», assimilée ici au nombre d'emplois à plein-temps dans le tertiaire. Chaque unité comprend la liste des communes les constituant. Nous avons par ailleurs jugé utile de faire figurer deux autres critères dans ce tableau. D'une part, ce que nous avons nommé «densité» et qui est le nombre d'emplois dans le tertiaire rapporté au nombre d'actifs du tertiaire qui y résident. Cet indice, dès lors qu'il est supérieur à 100 , implique que la zone «importe» plus d'actifs dans le tertiaire qu'elle n'en exporte et donc qu'elle est un centre d'emploi tertiaire. Nous avons également fait figurer sous «croissance» une indication de la croissance du nombre d'emplois tertiaires entre 1975 et 1995, exprimée en nombre d'emplois en 1995 pour 100 emplois vingt ans auparavant. La localisation et la taille de chaque Edge-City figure sur la carte 1, avec une indication du pourtour des agglomérations et des villes isolées (définition OFS de 1990) ainsi que de la position des axes autoroutiers du pays.

\subsection{Une répartition attendue?}

L'analyse de la carte 1 laisse apparaître plusieurs caractéristiques fondamentales du phénomène. En première lecture, il apparaît clairement que dans ses grandes lignes, il s'agit d'un phénomène périurbain, qui est d'autant plus fort que l'agglomération dans lequel il se produit est importante. Les cinq grandes villes du pays sont toutes escortées d'au moins une Edge-City. Le phénomène est particulièrement bien développé dans l'agglomération zurichoise, qui compte 8 unités regroupant près de 85000 emplois tertiaires. Genève (quatre unités regroupant 33000 emplois tertiaires), Bâle (trois Edge-Cities, 23000 emplois), et Lausanne (quatre unités, 21000 emplois) suivent d'assez loin la capitale économique du pays. Berne constitue un contre-exemple puisqu'elle ne compte qu'une Edge-City d'environ 7500 emplois. Il apparaît donc déjà que si la taille de l'agglomération-hôte joue un rôle, elle n'est pas seule en cause. Ceci se confirme lorsqu'on observe la répartition des autres EdgeCities du pays. Celles-ci ne sont liées que de manière marginale à la taille de l'agglomération qui les abritent. Fribourg, Lucerne ou Lugano connais- 


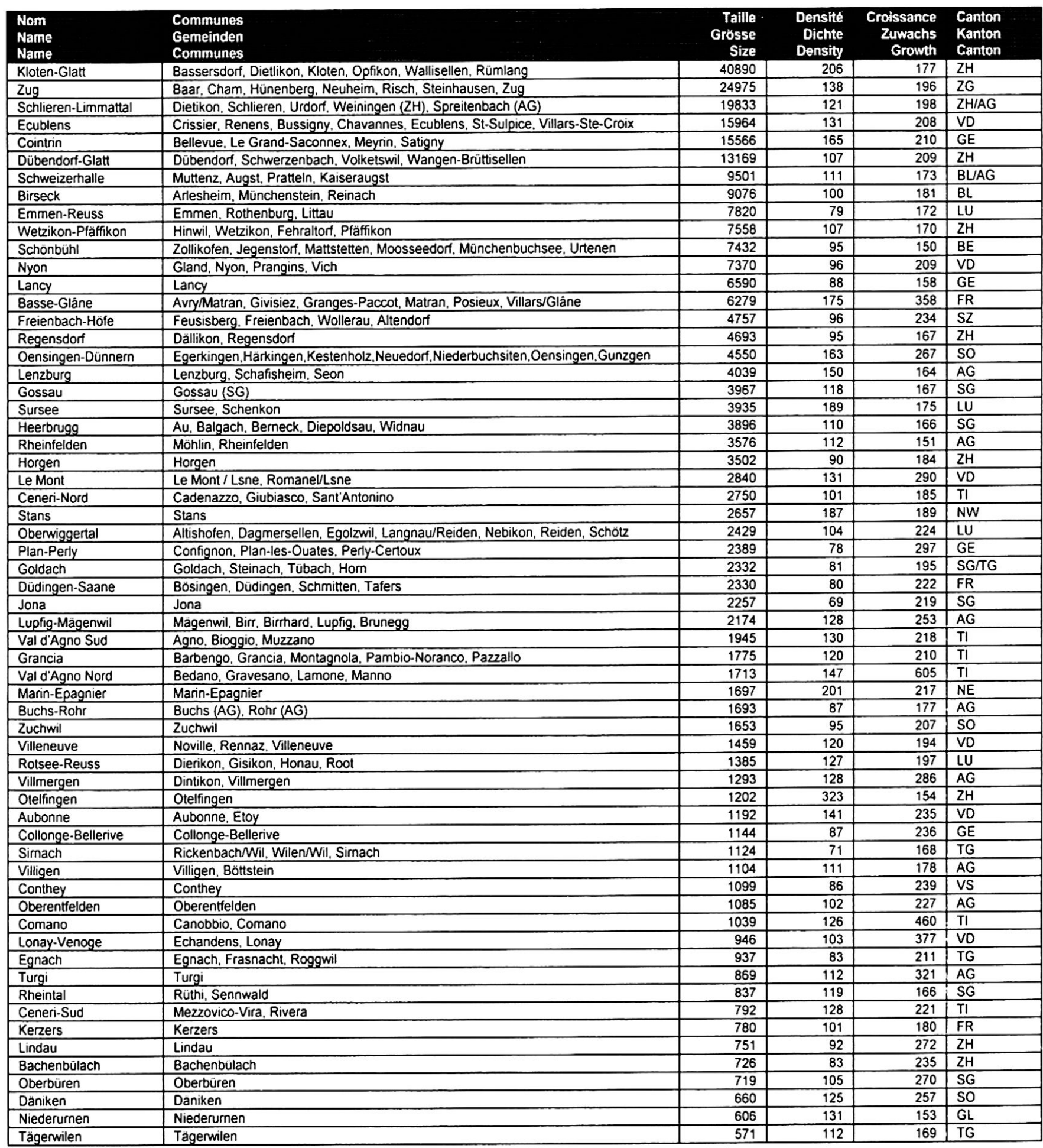

Tab. 2: $\quad$ Liste des Edge-Cities en Suisse

Listing of Switzerland's Edge Cities

Liste der Edge-Cities in der Schweiz

Taille: $\quad$ Nombre d'emplois à plein-temps dans le secteur tertiaire en 1995

Number of full-time work places in the service sector 1995

Zahl der Vollzeitarbeitsstellen im Dienstleistungssektor 1995

Densité: $\quad$ Nombre d'emplois dans le secteur tertiaire pour 100 résidents actifs dans le secteur tertiaire en 1995

Number of work places in the service sector for 100 residents working in service 1995

Zahl der Dienstleistungsarbeitsstellen für 100 im Dienstleistungssektor arbeitende Einwohner 1995

Croissance: Croissance du nombre d'emplois tertiaires entre 1975 et $1995(1975=100)$ (moyenne suisse: 124)

Growth of the number of work places in services between 1975 and $1995(1975=100)$ (Swiss average: 124)

Zuwachs der Dienstleistungsarbeitsstellen zwischen 1975 und 1995 (1975 = 100) (schweiz. Durchschnitt: 124) 
sent de grandes Edge-Cities (rapportées à leur taille), alors que des agglomérations de même taille, comme Bienne, Winterthour ou Thoune, n'en abritent pas.

Lorsqu'on observe le pays dans son ensemble, le Triangle d'Or ressort très nettement: la moitié des cas suisses s'inscrivent dans un triangle formé des agglomérations de Zurich, Bâle et Lucerne. Mieux encore, effet d'un contexte proprement métropolitain, les Edge-Cities du Triangle d'Or ne s'implantent pas uniquement en banlieue proche des grands centres : elles tendent à baliser tout ce territoire, souvent en s'appuyant sur un petit centre existant, comme à Rheinfelden, à Sursee, à WetzikonPfäffikon $\mathrm{ZH}$, à Lenzbourg. Le cas le plus spectaculaire de cette implantation dans un centre secondaire est celui de Zoug, dont toute l'agglomération se comporte comme une EdgeCity, en l'occurrence la seconde du pays avec 25000 emplois tertiaires. Si la majorité de ces Edge-Cities se situent dans des banlieues reconnues comme telles, certaines se situent en zone rurale, comme Sursee. D'autres connaissent un développement notable alors qu'elles ne s'appuient sur aucun centre important: ainsi Lupfig-Mägenwil, OensingenDünnern ou encore l'Oberwiggertal.

Ailleurs dans le pays, deux autres pôles semblent ressortir. L'arc lémanique compte dix Edge-Cities (dont neuf entre Lausanne et Genève), le Tessin en égrène six entre Bellinzone et Lugano. Les autres régions du pays sont moins touchées par le phénomène. La Suisse orientale compte huit EdgeCities largement dispersées et de petite taille, le Mittelland en compte six, les Alpes une (près de Sion), le Jura aucune.

\subsection{Le dictat des réseaux de communication}

Si la répartition des Edge-Cities souligne la position des grands centres du pays, tout en s'en écartant parfois de manière considérable, il est en revanche un paramètre qui semble jouer un rôle majeur, celui de l'accessibilité, et donc de la position par rapport aux axes de communication. La quasitotalité des Edge-Cities suisses s'organisent autour de jonctions autoroutières, ou à proximité immédiate de celles-ci. Par ailleurs, la bonne position par rapport aux aéroports internationaux joue un rôle majeur: Kloten est au centre de l'Edge-City la plus importante du pays, Cointrin en abrite une autre très importante, et à un moindre niveau, l'aérodrome d'Agno (Lugano). L'accessibilité par rapport au réseau autoroutier a également son importance à l'échelle de l'agglomération: les grandes EdgeCities zurichoises s'égrènent le long de l'autoroute d'évitement du centre, au nord de la ville; de même à Genève, à Lausanne, à Bâle, à Berne et pratiquement partout ailleurs: les parties des aggloméra- tions qui sont situées à l'écart des axes autoroutiers et de leurs jonctions ne jouissent pas du même développement.

Les Edge-Cities qui semblent se développer sans s'appuyer ni sur une agglomération importante, ni sur un centre secondaire s'adossent sur de grands nœuds autoroutiers du pays: c'est le cas d'OensingenDünnern (croisement A1-A2), de l'Oberwiggertal (croisement A1-A2 aussi) ou encore de LupfigMägenwil (croisement A1-A3). La plupart des grandes Edge-Cities du pays se situent en même temps à proximité d'un centre important et d'un croisement autoroutier: ainsi Ecublens au croisement A1-A9 dans l'ouest lausannois, Schönbühl au nœud A1-A6 au nord de Berne, Schweizerhalle au nœud A2-A3 à l'est de Bâle, Emmen au nœud A2-A14 au nord de Lucerne et bien sûr Kloten, Schlieren, Dübendorf à proximité des nœuds autoroutiers du grand Zurich.

La règle n'est toutefois pas absolue. D'une part, on trouve des nœuds autoroutiers qui n'ont pas donné lieu au développement d'Edge-Cities, comme par exemple au nord et au nord-est de Winterthour (A1-A4 et A1-A7), ou dans la Riviera vaudoise (A9-A12); à l'inverse, une Edge-City en tous cas, et non des moindres (Zoug), ne se développe pas autour d'un grand carrefour autoroutier. Il reste tout de même que bien plus que la taille et l'importance de l'agglomération-hôte, ce soit la position par rapport au réseau autoroutier et aux aéroports qui joue le plus grand rôle dans l'apparition et le développement des Edge-Cities. Ces lieux ont pour qualité d'être accessibles facilement.

\subsection{L'émergence du fait métropolitain}

Les deux premières étapes de notre analyse ont permis de mettre en exergue les facteurs qui président à l'apparition des Edge-Cities: proximité d'un grand centre, et surtout accessibilité. Des exceptions importantes demeurent: la ville de Berne est en même temps bien située et importante, mais elle ne développe pas d'Edge-Cities importantes. A l'inverse, Zoug n'est ni une grande ville, ni au croisement d'un carrefour stratégique, et pourtant elle constitue la seconde Edge-City de Suisse. Il y a donc d'importantes différences entre les régions de Suisse, qui ne semblent pas directement liées aux paramètres de position par rapport à un grand centre ou aux axes de communication.

Cette impression se renforce lorsqu'on observe les paramètres de densité et de croissance des EdgeCities. Nous avions constaté trois régions d'implantation préférentielle des Edge-Cities: le Triangle d'Or, l'arc lémanique et le Sottoceneri. Un examen plus serré laisse apparaître d'autres différences. Les emplois des Edge-Cities zurichoises, genevoises et lausannoises représentent environ $40 \%$ 
du nombre de ceux de leurs centres respectifs; à Bâle, ce chiffre est d'environ 30\%, à Berne il est inférieur à $10 \%$. Par ailleurs, les Edge-Cities bâloises et bernoises sont moins denses et se développent à un rythme moins soutenu que celles de l'arc lémanique ou de la région zurichoise (tabl. 1, illustration 1). On retrouve presque partout en Suisse une forte corrélation entre l'importance des EdgeCities par rapport à leur centre et leur «force» telle qu'elle s'exprime par la densité et le taux de croissance. Ainsi, certaines agglomérations sont fortement touchées: c'est le cas de Fribourg, de Lugano, sans parler évidemment des cas où la dynamique touche également le centre de l'agglomération, comme à Zoug, à Lenzbourg, à Wetzikon-Pfäffikon $\mathrm{ZH}$, à Pfäffikon-Lachen SZ. A l'inverse, les villes de Suisse orientale, voire du Plateau sont moins touchées, et le sont par des Edge-Cities moins vigoureuses. Tout se passe comme si, à l'échelle du territoire suisse, certaines régions étaient plus touchées que d'autres.

L'intérêt ici réside dans le fait que les régions les plus concernées correspondent aux métropoles. Il est reconnu depuis longtemps que le Triangle d'Or constitue de fait l'aire de la métropole zurichoise et que les diverses agglomérations qui le balisent, comme Zoug, Baden, Lenzbourg, Winterthour, entretiennent des liens de dépendance étroits avec Zurich. Or cette aire métropolitaine reconnue est à peu près celle qui englobe la concentration d'Edge-Cities du Triangle d'Or: tout se passe comme si les Edge-Cities étaient des marqueurs de métropoles. Ce lien métropolitain permet également d'expliquer la présence d'agglomérations entières se comportant comme des Edge-Cities, agglomérations qu'on ne trouve d'ailleurs que dans le voisinage zurichois et toutes reconnues comme faisant part la métropole; il explique aussi la présence d'Edge-Cities en l'absence de centres secondaires ou de banlieues, comme à Oensingen, Lupfig ou dans l'Oberwiggertal. Si l'on accepte cette vision, une bonne vingtaine d'Edge-Cities du Triangle d'Or peuvent se lire comme étant des Edge-Cities lointaines de la métropole zurichoise, elles et les agglomérations qu'elles semblent escorter (comme Zoug ou Lenzbourg).

A l'autre bout du pays, les Edge-Cities de l'axe Lausanne-Genève comptent près de 55000 emplois tertiaires; deux pôles de croissance sont en outre apparus le long de la Côte, l'un à $20 \mathrm{~km}$ de Lausanne (Aubonne), l'autre à $25 \mathrm{~km}$ de Genève (Nyon); c'est le seul endroit de Suisse en dehors du Triangle d'Or où un centre secondaire connaît une telle dynamique, le seul aussi où une petite localité «lointaine», en campagne, ressort autant. Il est frappant de constater que la zone concernée correspond à l'axe majeur de l'autre métropole suisse qui a été proposée, la métropole lémanique. Il est donc tentant de rattacher la dynamique des Edge-Cities à l'émergence des régions métropolitaines. Il nous apparaît même qu'il sera difficile de trouver une meilleure explication quant à la répartition spatiale des Edge-Cities de Suisse.

Pour autant, ce lien n'épuise pas entièrement le thème. A ce titre, les cas de Fribourg et surtout de Zoug, deux villes moyennes très touchées par le phénomène, montre bien que d'autres facteurs jouent encore un rôle tout à fait sensible. Ici, il semble bien que la politique fiscale (et la politique de place financière avec elle) joue un rôle majeur en Suisse. Après tout, les régions les plus touchées du pays correspondent aux places financières ou aux cantons fiscalement favorables aux entreprises: Zurich, Zoug, Genève, Lausanne, Lugano... et Fribourg.

\section{Une nouvelle Suisse urbaine?}

\subsection{Des aires métropolitaines qui s'affirment}

Il reste toutefois encore difficile de démêler l'écheveau des différents phénomènes qui président au développement des Edge-Cities. En particulier, les liens de causalité restent flous: il est par exemple difficile de savoir si les corridors autoroutiers ont favorisé le développement de certaines régions, ou si c'est le développement de ces régions qui a incité à la mise en place des autoroutes; on peut tenir le même raisonnement en ce qui concerne les «paradis fiscaux»: le développement économique entraînet-il l'assouplissement de la pression fiscale, ou est-ce le contraire? Quel est le lien entre métropolisation et apparition de "paradis fiscaux»? Ces questions sont centrales dans l'analyse de l'émergence des Edge-Cities. En effet, par exemple, si les autoroutes ou une politique fiscale favorable aux entreprises entraînent le développement de certaines régions, cela signifie que ces mesures ont un impact majeur sur le développement économique et surtout sur les différences régionales de développement. En ce sens, le développement finalement assez localisé des Edge-Cities ne ferait que traduire de manière visible les discriminations économiques entre les régions suisses, et marquer l'émergence d'aires métropolitaines économiquement fortes, «gagnantes», qui bien qu'étendues, ne concernent toutefois que certaines portions du territoire suisse.

Que signifie pour la Suisse l'apparition des EdgeCities? A l'échelle nationale d'abord, elles semblent donc être un excellent marqueur de développement économique. Le fait qu'elles n'émergent que dans certaines régions qui combinent urbanisation, accessibilité et souvent conditions fiscales favorables associées à la richesse intrinsèque locale 
implique que les différences de développement entre les régions suisses tendent à s'accroître: ce sont en effet les régions les mieux loties du pays qui bénéficient de cette nouvelle forme de développement urbain. En termes régionaux, loin de menacer les hiérarchies actuelles, le développement des EdgeCities indique un renforcement de cette hiérarchie et un accroissement des disparités régionales.

\subsection{Des centres classiques menacés}

A l'échelle des aires métropolitaines et des agglomérations ensuite, l'émergence des EdgeCities pose un sérieux problème économique, mais aussi politique. La montée en puissance des EdgeCities ne fait que traduire dans l'espace la suburbanisation croissante des emplois tertiaires, donc des emplois auparavant localisés dans les Central Business Districts. Bien sûr, les centre-villes conservent encore leur primauté, mais ils tendent à perdre de leur substance au profit de certaines de leurs banlieues et à terme, leur vitalité économique est menacée. Ceci pose un grave problème politique, car les disparités, déjà très inquiétantes comme le constate R. FREY (1997), entre centres stagnants et banlieues émergentes ne pourront alors que se renforcer, et par suite les conflits d'intérêts et les conflits politiques. Or, compte tenu de ce qui préside au développement des EdgeCities (en premier lieu, l'accessibilité et la commodité automobile), on voit mal comment les centres pourraient concurrencer les Edge-Cities, sans même parler de la politique des villes qui tend à restreindre l'accessibilité des centre-villes aux voitures. C'est dire que loin de contrecarrer cette disparité centre-banlieue, la politique urbaine actuelle la renforce de fait, et donc accompagne bien involontairement la perte de vitalité économique des centres. Dans ce contexte, il apparaît absolument nécessaire de mettre en place une politique des villes plus active et impliquant les banlieues, faute de quoi le fossé ne pourra que s'accroître entre un centre en voie de paupérisation et des banlieues de plus en plus autosuffisantes.

\subsection{Vers une urbanisation métropolitaine}

Le développement des Edge-Cities est un phénomène radicalement nouveau en ce sens que tout en restant un phénomène éminemment urbain, ces cités-lisières s'affranchissent presque totalement des centres traditionnels. Le développement actuel des activités dites centrales ne se fait plus dans les centres, mais à l'extérieur, en marge de ceux-ci, en s'appuyant beaucoup plus sur l'accessibilité que sur la centralité, en s'étalant et en se dispersant sur le territoire. Ceci implique une nouvelle définition du phénomène urbain. L'urbanisation suisse ne s'appuie plus sur les centres historiques: elle touche désormais de grandes aires métropolitaines, et au sein de ces aires, la hiérarchie entre les pôles tend à se dissoudre, certaines localités vivant une véritable explosion économique et démographique alors que d'autres stagnent, sans que la hiérarchie préexistante ne joue aucun rôle. En 1995, la hiérarchie urbaine suisse vue dans cette perspective est déjà bouleversée: avec ses 40000 places de travail, l'Edge-City de Kloten-Glatt est le sixième centre d'emploi tertiaire du pays, talonnant Lausanne et ayant dépassé Lucerne ou St-Gall. Au-delà de certaines permanences, la carte de la Suisse urbaine se recompose complètement. Au sein des aires métropolitaines, les hiérarchies classiques sont battues en brèche par l'émergence de lieux nouveaux, promis à un brillant avenir et que la science géographique a tout intérêt à suivre de près: les EdgeCities sont peut-être les centres économiques de la Suisse de demain.

\section{Bibliographie}

Bassand, M., Leresche, J. Ph. \& D. Joye (1995): Métropolisations, interdépendances mondiales et implications lémaniques. Genève.

BERRY, B. J.L. \& H. M. KIM (1993): Challenges to the monocentric model. - In: Geographical Analysis, 25, 1: 1-4.

Dessemontet, P. \& J.B. Racine (1996): Villes et agglomérations suisses. - In: Geographica Helvetica, 4/1996: 143-68.

FREY, R. (1997): Städtische Wirtschaft, Mobilität und Umwelt. - In Collectif: «Die Stadtmorgen», Union des Villes Suisses: 19-26.

Garreau, J. (1991): Edge-city: life on the new frontier. New-York.

SCHUler, M. (1994): Les niveaux géographiques de la Suisse, OFS, Berne.

VANCE, J. (1990): The continuing city: urban morphology in western civilization. Baltimore.

\section{Résumé: Des Edge-Cities en Suisse? \\ L'émergence de nouveaux pôles d'activités métropolitaines sur le territoire helvétique}

Depuis 20 ans, certaines banlieues de Suisse s'affirment en tant que centres d'emploi tertiaire, à l'instar des Edge-Cities nord-américaines. Le but de cette contribution est de faire le point sur la situation en 1995. Après un bref rappel historique, cette étude propose une méthode statistique de détection du phénomène, qui s'appuie notamment sur les paramètres de croissance du secteur tertiaire, ainsi que sur l'affirmation de ces lieux en tant que centres d'emploi tertiaire. En appliquant cette méthode aux communes suisses, 171 communes formant 61 unités de plus de 500 emplois sont 
détectées à travers le pays. Ces «Edge-Cities» suisses sont particulièrement importantes aux abords des centres majeurs du pays, comme Zurich et Genève, mais elles colonisent également les espaces métropolitains, suivant les lignes de pénétration que représentent les autoroutes, et délaissant en revanche les régions périphériques. Leur émergence souligne d'une part que les disparités régionales semblent se renforcer, et d'autre part que les centres classiques sont désormais concurrencés par des localisations d'un nouveau style. A ce titre, les «Edge-Cities» suisses, à leur échelle, représentent une problématique nouvelle pour les géographes.

\section{Summary: Edge-Cities in Switzerland? \\ The emergence of new metropolitan employment centers on Swiss territory}

Since the 1970's, some Swiss suburbs have been rising as new centers of services and employment, very similar to American Edge-Cities. The objective of this paper is to draw a picture of this phenomenon as of 1995. After a brief historical background, this study offers a statistical method of detecting this phenomenon, based on parameters such as growth of tertiary activities and emergence of such places as service centers. Applying this method to Swiss communes, 171 communes grouped into 61 units with more than 500 service jobs have been identified throughout the country. Swiss Edge-Cities are highly developed in the suburbs of major urban areas. They also colonize metropolitan areas, following penetration lines represented by highways, thus ignoring peripherial regions. The emergence of edge cities, albeit on a much smaller scale than in the US, reveals that regional disparities have intensified and that core cities are now being challenged by new suburban locations. At their own and much smaller scale, therefore, Swiss "EdgeCities" represent a new area of research for geographers.

\section{Zusammenfassung: Edge-Cities in der Schweiz? \\ Das Auftreten neuer suburbaner \\ Wirtschaftszentren in der Schweiz}

Seit 20 Jahren spielen einige Vorstädte in der Schweiz als Zentren für die Dienstleistungswirtschaft eine wichtige Rolle, vergleichbar den Edge-Cities in Nordamerika. Das Ziel dieses Artikels ist, eine Bestandsaufnahme der Situation im Jahre $1995 \mathrm{zu}$ machen. Nach einem kurzen historischen Überblick schlägt diese Studie eine statistische Erkennungsmethode des Phänomens vor, die sich u.a. auf Zuwachsparameter der Dienstleistungswirtschaft stützt, sowie auf die Rolle dieser Orte als Dienstleistungszentren. Die Anwendung dieser Methode auf Schweizer Gemeinden identifizierte 171 Gemeinden, die 61 Ein- heiten von mehr als 500 Arbeitsplätzen bilden. Diese Schweizer «Edge-Cities» sind besonders in den Randgebieten der grössten urbanen Zentren, wie Zürich oder Genf, entwickelt. Sie kolonisieren jedoch auch Metropolitanräume, und folgen dabei den von den Autobahnen dargestellten Durchdringungslinien und ignorieren somit die Randregionen. Ihr Auftreten zeigt, dass die regionalen Ungleichheiten stärker werden und dass die klassischen Innenstadtzentren andererseits von nun an mit Standorten neuer Art konkurrieren müssen. In diesem Rahmen stellen die Schweizer «EdgeCities» in ihrem Massstab eine neue Fragestellung für die Geographen dar.
Pierre Dessemontet, géographe dipl., Institut de Géographie, Université de Lausanne, BFSH II-Dorigny, $\mathrm{CH}$-Lausanne; Directeur Développement et Services auprès de MicroGIS Geomatics, SA, Rue des Jordils 40, CH-1025 St-Sulpice. 